\title{
Ultraviolet Line-Continuum properties of Seyfert 1 galaxies and quasars
}

\author{
Vijayakumar H. Doddamani and P. Vedavathi \\ Dept. of Physics, Bangalore University, Bangalore-560056, India \\ email: drvkdmani@gmail.com
}

\begin{abstract}
Active galaxies are most luminous objects in the universe whose spectra are characterized by both permitted and forbidden emission line features. The spectra of Seyfert 1 galaxies and quasars contain strong and broad emission lines of wide ranging ionization potentials. The velocity widths of the lines range from a minimum of $\approx 500 \mathrm{~km} / \mathrm{s}$ for narrow lines to a maximum of $20,000 \mathrm{~km} / \mathrm{sec}$ for broad lines. The UV spectra of the active galaxies contain strong and broad emission lines such as Ly $\alpha, \mathrm{NV}$, SiIV, OIV], CIV, CIII] and MgII lines. The widths of the broad lines are attributed to the differential doppler shifts of the emission lines due to the bulk motions of individual clumpy gas clouds in the BLR region. We have anlysed UV spectra of Seyfert 1 galaxies and quasars from IUE satellite archival database to understand the nature of dependence of the emission line properties with the underlying UV continuum. We have undertaken line luminosity correlation studies for Ly $\alpha$ and CIV lines with their underlying UV continuum luminosity at $1125 \AA, 1425 \AA \& 1625 \AA$. The IUE archival spectra have been reduced for galactic reddening using the $\mathrm{E}(\mathrm{B}-\mathrm{V})$ and $\mathrm{NHI}$ values published continuum luminosity has been observed at $1125 \AA, 1425 \AA \& 1625 \AA$. The Ly $\alpha$ line line has exhibited strongest linear correlation wavelengths while CIV line has shown at $1425 \AA$ and $2625 \AA$ wavelengths. These results are empirically consistent with the predictions of the general multi-component photo-ionization models suggesting that the central strong UV continuum has been reprocessed by the clumpy gas clouds of the broad emission region (BLR). A detailed account of the data reduction, UV flux measurement and the significance of line-luminosity correlations are discussed in this paper.
\end{abstract}

Keywords. Active galaxies, Seyfert 1 galaxies, quasars, continuum and line luminosity, black hole

\section{Introduction}

The Active galactic Nuclei (AGNs) emit radiation over the entire electromagnetic spectrum from the highest energy $\gamma$-photons to the lowest energy radio waves. To a first order approximation the continua of AGNs are similar over seven orders of magnitude in wavelengths. Active galaxies are most luminous objects in the universe whose spectra are characterized by both permitted and forbidden emission line features. The spectra of Seyfert 1 galaxies (Sy1) and quasars contain strong and broad emission lines of wide ranging ionization potentials. The velocity widths of the lines range from a minimum of $\approx 500 \mathrm{~km} / \mathrm{s}$ for narrow lines to a maximum of $20,000 \mathrm{~km} / \mathrm{sec}$ for broad lines. The Ultra Violet spectra of these active galaxies contain strong and broad emission lines such as Ly $\alpha$, NV, SiIV, OIV], CIV, CIII] and MgII lines. The widths of the broad lines are attributed to the differential Doppler shifts of the emission lines due to the bulk motions of individual clumpy clouds. Thus, it becomes very much interesting to study the dependence of emission line parameters with the underlying UV continuum in Sefert1 galaxies and quasars with the advent UV spectroscopic observations by IUE satellite (Bogess A et al. 1978). It is widely believed that the strong UV lines are emitted by the re-processing of the strong UV continuum radiation by the clumpy gas clouds of BLR. 

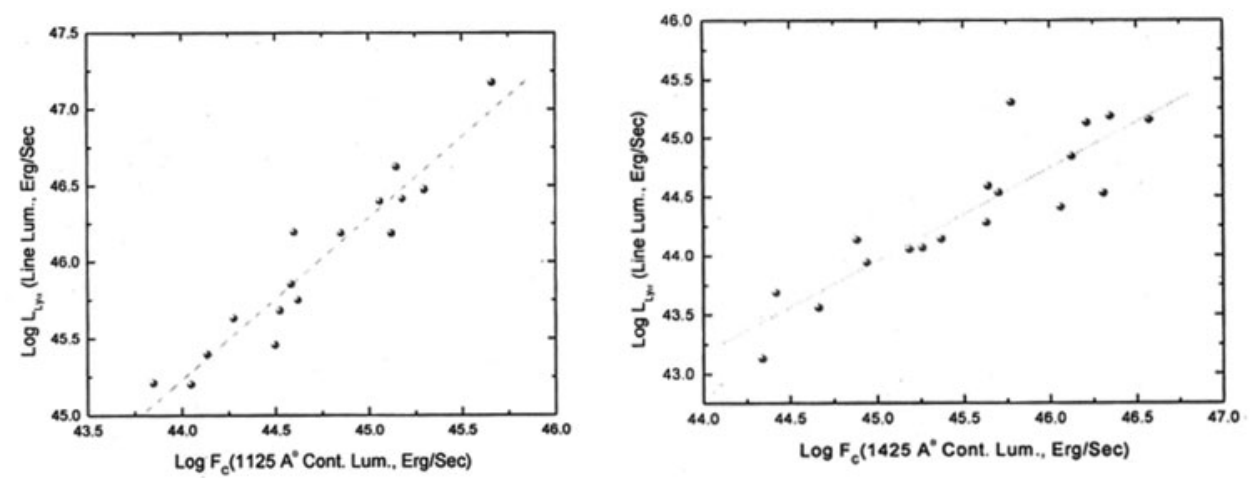

Figure 1. Dependence of Ly-alpha line luminosity with UV continuum luminosity at $1125 \AA$ and $1425 \AA$.
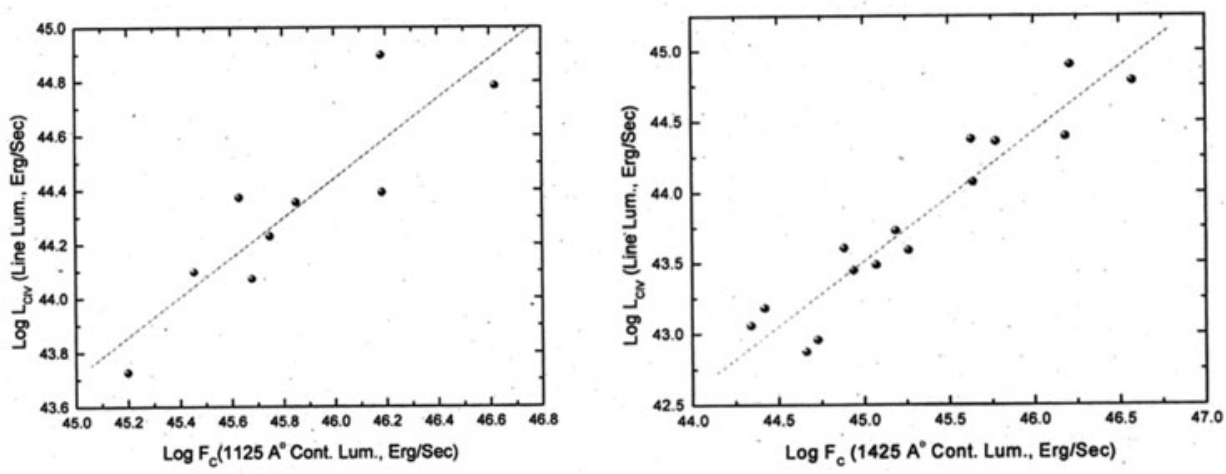

Figure 2. Correlation of CIV line luminosity with $1125 \AA$ and $1625 \AA$ continuum luminosity.

\section{IUE Data Analysis}

The flux calibrated IUE spectra have been reduced for galactic reddening using published E(B-V) values from Obrien et al.(1988), Kinney A L et al.(1991), Felix J Lockman (1995) and David J Schelgel (1998) papers. The spectral data reduction have been carried out using the dered and dopcor tasks of IRAF. We have measured the continuum fluxes at $1125 \AA, 1425 \AA$ and $1625 \AA$ rest wavelengths as we found that these continuum windows to be sufficiently away from the wavelengths of the emission lines. We have measured the continuum fluxes with $50 \AA$ wide continuum window by sampling two wavelength values centered about the above mentioned continuum wavelengths. The continuum measurement task of IRAF gives the mean continuum flux value over the $50 \AA$ wide window and the rms deviation of the measurement flux from the mean flux, which is considered as the most probable uncertainty in the continuum flux measurements. The signal to noise ratio (SNR) of the IUE camera ranges between $\approx 3-40$ and we have selected the continuum measurements for which the $\mathrm{SNR} \geqslant 5$. We have calculated the mean value of the continuum flux in case of the repeated observations. The study of AGNs has drawn overwhelming attention because of its extraordinary luminosity emitted by the matter accretion processes taking place at the central super massive black hole. The luminosity of AGNs typically lies in the $10^{42}-10^{48} \mathrm{erg} / \mathrm{sec}$ range, thus making these objects as the highest luminosity objects in the universe. We have calculated the luminosity of Sy1 galaxies and quasars using the relation 


$$
L_{\lambda}=4 \Pi D_{L}^{2} F_{\lambda} \mathrm{erg} / \mathrm{sec},
$$

where $\mathrm{L}_{\lambda}$ is the monochromatic luminosity at wavelength $\lambda \mathrm{D}_{L}$ is the luminosity distance of the object and $\mathrm{F}_{\lambda}$ is the monochromatic flux. The luminosity-distances $\left(\mathrm{D}_{L}, \mathrm{Mpc}\right)$ of the objects have been calculated using the luminosity-distance equation of Terrell (1977) with $\mathrm{H}_{0}=70 \mathrm{Km} / \mathrm{Sec} / \mathrm{Mpc}$. To explore the nature of the dependence the luminosity of Ly $\alpha$ and CIV lines with the underlying UV continuum luminosity at $1125 \AA, 1425 \&$ $2625 \AA$, we have carried out the correlation study of line luminosity with the underlying continuum since a strong direct dependence has been observed in the optical lines and their underlying optical non-thermal continuum.

\section{Results and discussions}

We see from figures 1 and 2 that Ly $\alpha$ exhibits the most tight correlation at $1125 \AA$, $1425 \AA$ continuum wavelengths with a mean $\mathrm{R}=0.939$ and CIV line with $\mathrm{R}=0.893$ at $1425 \AA$ and $2625 \AA$. Thus we see a similarity in the dependence of low and high ionization lines by the strong UV continuum emitted by the central accretion disk of AGNs. The UV spectroscopy of Sy 1 galaxies and quasars has established the similarity of Seyfert 1 and quasars in terms of their UV line and continuum properties. These results would help us to unify the Seyfert 1 galaxies and quasars. Further, it could also be concluded that our results are empirically consistent with the predictions of the general photo-ionization models, suggesting that the central strong continuum would have been re-processed by the clumpy gas clouds of broad emission line (BLR) region. We feel that these results could become significant for the construction/constraining of BLR models.

\section{Acknowledgements}

This work has been carried out under a UGC major research project sanctioned to the first author. Authors would like to acknowledge the help extended by the Scientific and Technical staff of Indian Institute of Astrophysics, Bangalore in carrying out this work. The cooperation extended by the Chairman, Department of Physics, Bangalore University, Bangalore towards the implementation of the project successfully is specially acknowledged. I also acknowledge support of the Department of Science and Technology, UGC, New Delhi, for its financial support.

\section{References}

Bogess, A. et al. 1978, Nature, 275, $372 \& 377$

Schelgel, D. J. 1998, ApJ 500, 525

Murphy, E. M. 1996, ApJ 105, 369

Lockman, F. J. 1995, ApJ 302, 342

Kinney, A. L. 1999, ApJS 74, 347

O'Brien, P. T. et al. 1998, MNRAS 233,845

Doddamani, Vijayakumar. H., Anandram, M. N., \& Ghosh, K. K. 2001, in: UV Emission line studies of ESOB113 and MRK150, In the Proceedings of the International Conference on "Multicolour Universe" held at TIFR, Mumbai, 11-15, Sep 2001, p. 161 